\title{
Failed reinnervation in aging skeletal muscle
}

Sudhakar Aare ${ }^{1,2}$, Sally Spendiff ${ }^{1,2}$, Madhusudanarao Vuda ${ }^{1,2}$, Daren Elkrief ${ }^{1,2,3}$, Anna Perez ${ }^{1}$, Qinghua Wu ${ }^{1,2}$, Dominique Mayaki', Sabah N. A. Hussain', Stefan Hettwer ${ }^{4}$ and Russell T. Hepple 1,2,3* $^{1}$

\begin{abstract}
Background: Skeletal muscle displays a marked accumulation of denervated myofibers at advanced age, which coincides with an acceleration of muscle atrophy.
\end{abstract}

Methods: In this study, we evaluated the hypothesis that the accumulation of denervated myofibers in advanced age is due to failed reinnervation by examining muscle from young adult (YA) and very old (VO) rats and from a murine model of sporadic denervation secondary to neurotrypsin over-expression (Sarco mouse).

Results: Both aging rat muscle and Sarco mouse muscle exhibited marked fiber-type grouping, consistent with repeating cycles of denervation and reinnervation, yet in VO muscle, rapsyn at the endplate increased and was associated with only a $10 \%$ decline in acetylcholine receptor (AChR) intensity, whereas in Sarco mice, there was a decline in rapsyn and a $25 \%$ decrease in AChR intensity. Transcripts of muscle-specific kinase (21-fold), acetylcholine receptor subunits a (68-fold), $\varepsilon$ (threefold) and $\gamma$ (47-fold), neural cell adhesion molecule (66-fold), and runt-related transcription factor 1 (33-fold) were upregulated in VO muscle of the rat, consistent with the marked persistent denervation evidenced by a large proportion of very small fibers (>20\%). In the Sarco mice, there were much smaller increases in denervation transcripts (0-3.5-fold) and accumulation of very small fibers (2-6\%) compared to the VO rat, suggesting a reduced capacity for reinnervation in aging muscle. Despite the marked persistent denervation in the $\mathrm{VO}$ rat muscle, transcripts of neurotrophins involved in promoting axonal sprouting following denervation exhibited no increase, and several miRNAs predicted to suppress neurotrophins were elevated in VO rat.

Conclusions: Our results support the hypothesis that the accumulation of denervated fibers with aging is due to failed reinnervation and that this may be affected by a limited neurotrophin response that mediates axonal sprouting following denervation.

Keywords: Aging, Skeletal muscle, Denervation, Neurotrophins, MicroRNA, Muscle atrophy, Sarcopenia, Reinnervation

Abbreviations: AChR, Acetylcholine Receptor; BDNF, Brain-derived Neurotrophic Factor; CNTF, Ciliary Neurotrophic Factor; CNTFR, CNTF Receptor; CPLA2, Cytoplasmic Phospholipase A2; GDNF, Glial Derived Neurotrophic Factor; GFRA1, GDNF Family Receptor Alpha-1; MHC, Myosin Heavy Chain; miRNA, MicroRNA; MuSK, Muscle Specific Kinase; Nav ${ }_{1.5}$, Sodium Channel Isoform 1.5; NCAM, Neural Cell Adhesion Molecule; NGF, Nerve Growth Factor; NMJ, Neuromuscular Junction; NTRK1, Neurotrophic Tyrosine Kinase Receptor 1; NTRK2, Neurotrophic Tyrosine Kinase Receptor 2; NTRK3, Neurotrophic Tyrosine Kinase Receptor Type 3; p75NTR, p75 Neurotrophin Receptor; phospho-MuSK, Phosphorylated MuSK; RUNX1, Runt-related Transcription Factor; Sarco, Neurotrypsin Over-expressing Mouse; UTR, Untranslated Region; VL, Vastus Lateralis; VO, Very Old; WT, Wild Type; YA, Young Adult

\footnotetext{
* Correspondence: Russell.hepple@mcgill.ca

${ }^{1}$ Research Institute of the McGill University Health Centre, EM2.2232,

RI MUHC, 1001 Decarie Blvd, Montreal, QC, Canada H4A 3J1

${ }^{2}$ McGill Research Centre for Physical Activity and Health, McGill University,

Montreal, QC, Canada

Full list of author information is available at the end of the article
} 


\section{Background}

Skeletal muscle undergoes repeating cycles of denervation and reinnervation for much of adult life [1]. However, in very advanced age, when muscle atrophy becomes severe and has clinical impact in terms of impairing mobility [2], there is a marked accumulation of persistently denervated muscle fibers $[3,4]$. Notably, we have shown in very old (VO) rat muscle that denervated myofibers are on average 35-50\% smaller than innervated fibers and innervated fibers of VO muscle are only $7 \%$ smaller than in young adult [3]. The significance of this is that denervation is the primary cause of atrophy in the period of the lifespan when the clinical impact of aging muscle is most relevant. As an accumulation of persistently denervated fibers marks the acceleration of atrophy in both slow and fast twitch muscles with aging [5], understanding the mechanisms contributing to their accumulation is essential to provide effective targets for combating age-related muscle atrophy.

Although there have been a number of recent studies examining the mechanisms that may contribute to destabilization of the aging neuromuscular junction (NMJ) and subsequently lead to denervation of muscle endplates [6-8], there is scant information about the potential mechanisms contributing to impaired reinnervation of denervated fibers in very advanced age. Importantly, the progressive increase in fiber type grouping seen with increasing age [9] is clear evidence that following a sporadic denervation event, such fibers are usually successfully reinnervated for much of the lifespan. For this reason, a likely cause for the accumulation of persistently denervated fibers in very advanced age is a failure of reinnervation. In this context, previous studies have noted an impaired reinnervation response following acute nerve injury in aged animals that is due to impaired axonal regrowth $[10,11]$. Despite this evidence, no prior studies have considered whether this impaired capacity for axonal regrowth (identified following acute nerve injury in aged animals) might also contribute to the accumulation of persistently denervated muscle fibers following denervation events that are part of the normal process of aging.

In addressing factors that might cause a compromised reinnervation response with aging, it is relevant to note that following surgical denervation in healthy young animals, there is a robust increase in neurotrophins, including brain-derived neurotrophic factor (BDNF) [12]. Furthermore, forced over-expression of the neurotrophins BDNF, glial-derived neurotrophic factor (GDNF) and nerve growth factor (NGF) in muscle facilitate axonal sprouting and reinnervation following surgical denervation [13]. Similarly, the exercise-induced enhancement of reinnervation following nerve crush injury is dependent upon muscle BDNF signaling [14]. As such, examination of neurotrophins involved in reinnervation and microRNAs (miRNAs) predicted to suppress neurotrophins could provide insights to the potential cause of failed reinnervation in very advanced age or at the least identify whether this pathway might represent a therapeutic target.

To help address these issues, we examined muscle morphology (fiber type and size distribution, fiber type grouping) and denervation-related transcripts (e.g., muscle specific kinase [MuSK], AChR subunits, cytoplasmic phospholipase A2 [cPLA2], neural cell adhesion molecule [NCAM], and runt-related transcription factor 1 [RUNX1]) in vastus lateralis (VL) muscle from young adult (YA; 8 months old) and very old (VO; 35 months old) male Fisher $344 \times$ Brown Norway F1-hybrid (F344BN) rats and compared this to the same measures made in soleus and gastrocnemius muscles from male wild-type (WT) and neurotrypsin-over-expressing mice (Sarco mouse; a model of sporadic denervation). A key element of the study design is that the age of the Sarco mice ( 8 months of age) allows us to evaluate the capacity for reinnervation of young adult muscle subjected to sporadic denervation. We hypothesized that both VO rats and Sarco mice would exhibit marked fiber type grouping (indicating repeating cycles of denervation and reinnervation in both models) but compared to Sarco mice, VO rat muscle would exhibit a greater accumulation of small fibers indicative of persistent sporadic denervation and a commensurately greater expression of denervation-related transcripts. As both aging [9] and neurotrypsin over-expression [7] cause repeating cycles of denervation and reinnervation, a greater accumulation of sporadically denervated muscle fibers and up-regulation of denervation-related transcripts would implicate failed reinnervation as a major contributor to the accumulation of persistently denervated muscle fibers in advanced age. To provide insights to potential mechanisms of failed reinnervation in VO muscle, we also examined neurotrophin transcripts and miRNAs predicted to blunt neurotrophin levels.

\section{Results \\ Fiber type proportions and fiber type grouping in aged rat and Sarco mice}

Figure 1a shows representative images of the rat vastus lateralis and Sarco mouse soleus muscle cross-sections labeled using primary antibodies against myosin heavy chain (MHC) isoforms to identify fiber type and laminin to distinguish fiber borders. Proportions of pure fibers (types I, IIa, IIb, and IIx) were lower in the VO rat muscle compared to YA and lower in Sarco mice than wild-type mice. This decline in pure fibers was accounted for by an increase in abundance of fibers co-expressing two or more MHCs (MHC co-expressing fibers) in the VO rat and Sarco mice (Fig. 1b), a phenotype that we have shown previously to be largely secondary to denervation in the $\mathrm{VO}$ rat muscle [3]. To obtain an index of the occurrence of denervation-reinnervation events, we examined the abundance of fibers totally enclosed by fibers of the same type 


\section{a}
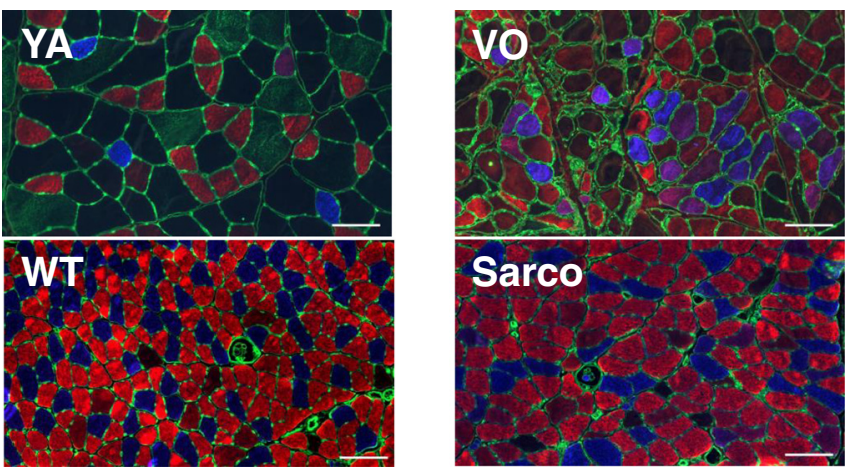

b
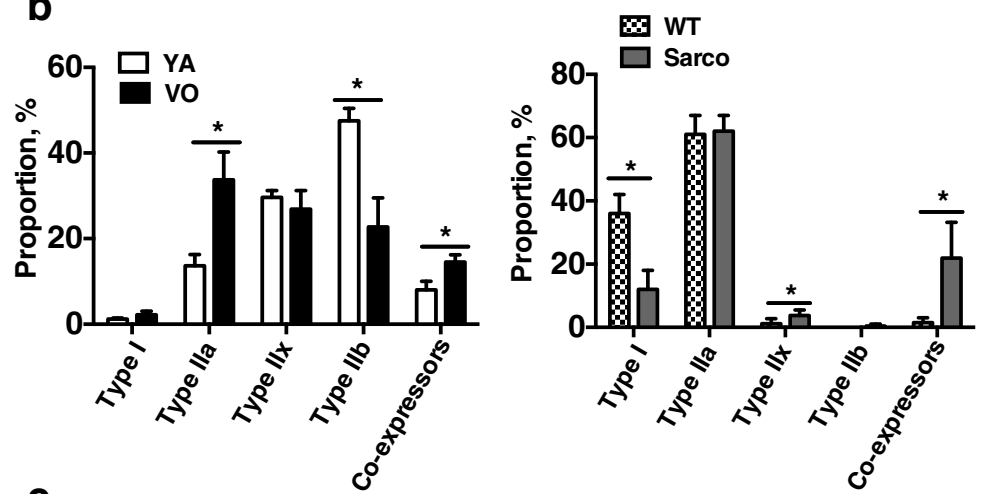

C
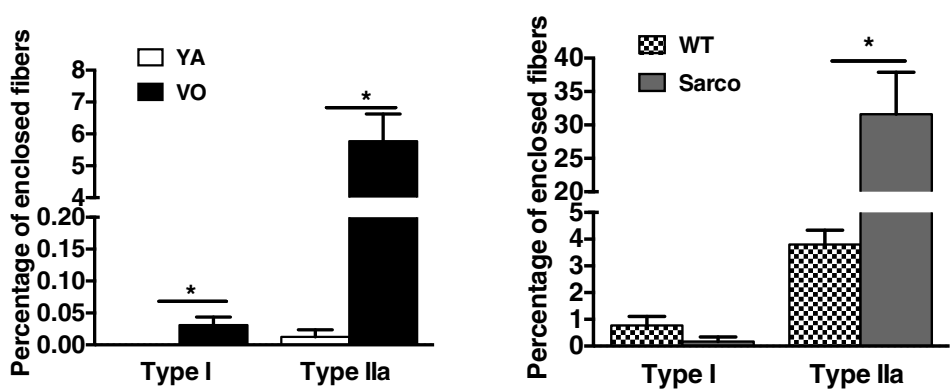

Fig. 1 Muscle fiber type and type grouping. a Transverse muscle sections from rat vastus lateralis (VL) and mouse soleus muscle. Young adult (YA) vs very old (VO) rat VL muscle and wild-type (WT) vs Sarco mouse soleus were labeled with laminin to visualize the basal lamina and antibodies against the myosin heavy chains to visualize fiber types. Scale bars = $100 \mu \mathrm{m}$. Type I (blue), type Ila (red), type IIx (green), type IIb (black [no staining]) were labeled. $\mathbf{b}$ Fiber type proportions in YA vs VO rat and WT vs Sarco mouse. c Fiber type grouping in YA vs VO rat and WT vs Sarco mouse. In $\mathbf{b}$ and $\mathbf{c}$, the data shown are means with standard error

(fiber type grouping) in rat and mouse muscle. This analysis revealed that there was a large increase in fiber type grouping in both VO rat and Sarco mouse muscle (Fig. 1c).

\section{MuSK-rapsyn at the neuromuscular junction}

To evaluate changes in one of the primary signaling axes for regulating the integrity of the post-synaptic AChR plaque, we examined phospho-MuSK, rapsyn, and AChR intensity levels in the endplate region of the muscle fiber in both the aging and Sarco mice (Fig. 2). Interestingly, whereas immunofluorescent images show relatively preserved phospho-MuSK and semi-quantitative analysis in muscle cross-sections revealed an increase in rapsyn and only a small (10\%) decline in AChR intensity in VO rat muscle, phospho-MuSK appeared considerably reduced in Sarco Soleus muscle and this was accompanied by a decline in rapsyn and relatively large $(25 \%)$ decline in AChR intensity.

\section{Fiber size in aged rat and Sarco mice}

To gain insight into the frequency of failed reinnervation events in the VO rat, we examined the abundance of muscle fibers exhibiting a size of $\leq 1000 \mu \mathrm{m}^{2}$, as these 


\section{a}

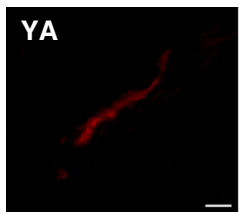

VO

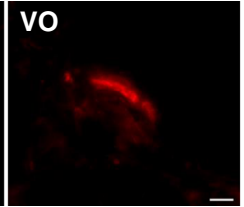

b

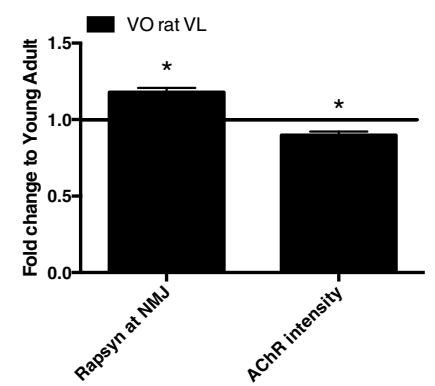

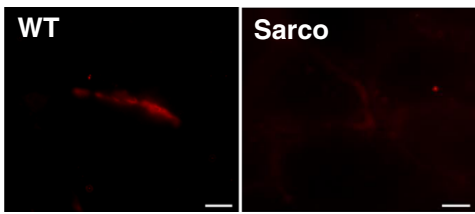

\section{Sarco}

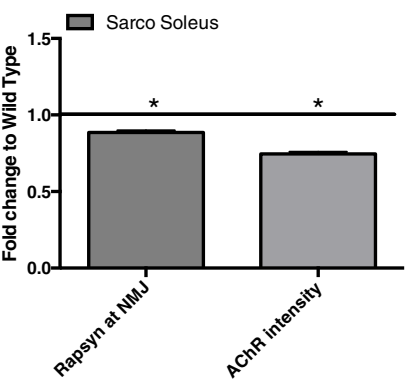

Fig. 2 Protein analyses at the muscle endplate region. a Representative images of muscle endplates from YA vs VO (VL muscle) and WT vs Sarco (gastrocnemius muscle) labeled with primary antibodies against phospho-MuSK (scale bars $=10 \mu \mathrm{m}$ ). $\mathbf{b}$ Semi-quantitative analysis of protein intensities at the muscle endplate for rapsyn and AChRs in YA ( $n=154-158$ endplates) vs VO ( $n=304-311$ endplates) (VL muscle) and WT ( $n=185$ endplates) vs Sarco ( $n=287-296$ endplates) (gastrocnemius muscle). Values are normalized as a fold change of YA or WT, respectively. ${ }^{*} P<0.05$ vs corresponding group within the same species

represent a population of muscle fibers shown by our group in VO rat gastrocnemius muscle to exhibit a high association with positive labeling for a marker of denervation $(>90 \%$ of these fibers are positive for the sodium channel isoform, $\mathrm{Nav}_{1.5}$ ) and to represent $<1 \%$ of fibers from young adult muscle [3]. We then compared this to a corresponding fiber size in Sarco mice $\left(650 \mu \mathrm{m}^{2}\right.$ in gastrocnemius, $575 \mu^{2}$ in soleus), based upon these representing a similar percentile of fiber size in the WT mice as in the YA rat. Interestingly, whereas $\mathrm{VO}$ rat exhibited $>20 \%$ of these very small fibers, Sarco mice exhibited approximately $2-6 \%$ of these very small fibers (higher in soleus than gastrocnemius) (Fig. 3a). Taken in the context of the high frequency of denervationreinnervation events evidenced by the large increase in fiber type grouping in both VO rat VL and Sarco mouse soleus muscle noted above (Fig. 1c), our analysis of very small fiber abundance reveals a marked impairment in reinnervation in $\mathrm{VO}$ rat muscle. On the other hand, a degree of compensatory hypertrophy was visually evident in photomicrographs of Sarco soleus muscle (Fig. 1a), consistent with a previous report [7].

\section{Transcriptional profile of denervation in aged rat and Sarco mice}

The expression of denervation-related transcripts was dramatically increased in VL muscle of VO rat samples when compared to YA, and these increases were markedly greater than seen in Sarco mouse soleus or gastrocnemius muscles (Fig. 3b). Specifically, transcript levels of MuSK, a key signaling molecule involved in regulation of AChR clustering at the neuromuscular junction $[15,16]$, increased 21 -fold in VO muscle (20-50 \% change in Sarco mouse muscles). Similarly, transcript levels of the $A C h R$ subunit $\alpha$ (68-fold in VO vs YA) and $\gamma$ (47-fold in $\mathrm{VO}$ vs $\mathrm{YA}$ ) increased to a much greater degree in $\mathrm{VO}$ rat muscle than in Sarco mouse soleus muscle where $A C h R \alpha$ had a borderline significant increase and $A C h R \gamma$ modestly increased (threefold) (no change in gastrocnemius muscle) vs wild-type mice. Interestingly, whereas $\mathrm{AChR} \varepsilon$ increased approximately threefold in VO rat VL muscle, it declined by nearly $60 \%$ in Sarco soleus muscle. Transcript levels of NCAM (50-fold higher in VO vs YA), which encodes a protein that increases in aging muscle [17] and is involved in restoration of nerve contact to the muscle [18], and RUNX1 (40-fold higher in VO vs YA), which encodes a protein that also increases in aging muscle [19] and counters muscle atrophy [20], were markedly elevated in VO rat muscle but not in Sarco mouse soleus or gastrocnemius muscle. Transcript levels of $c P L A_{2}$, an enzyme catalyzing release of mitochondrial lipids as fatty acid hydroperoxides following denervation [21], increased in VO rat VL muscle and in Sarco mouse Soleus muscle (both approximately twofold relative to their respective controls). Collectively, these results identify a markedly greater denervation-related transcriptional profile in $\mathrm{VO}$ rat muscle than the Sarco mouse, despite both models being subjected to recurring cycles of sporadic denervation. 
a
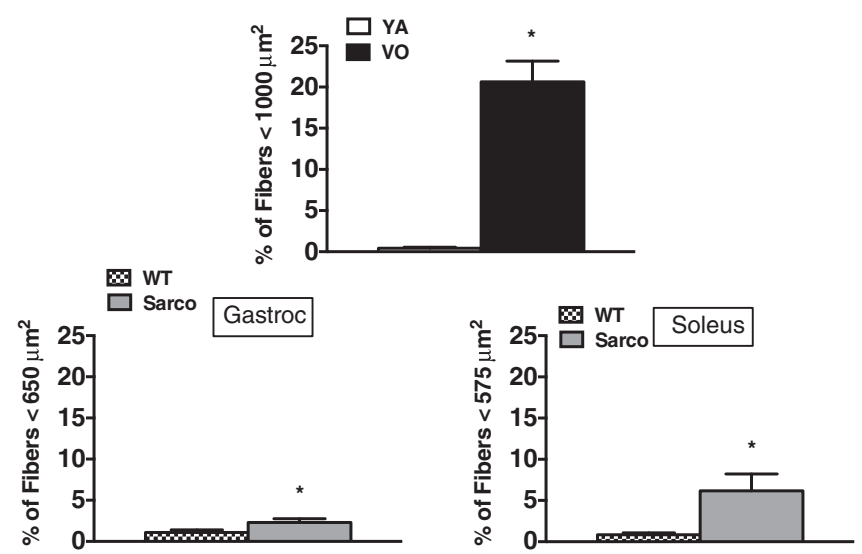

b
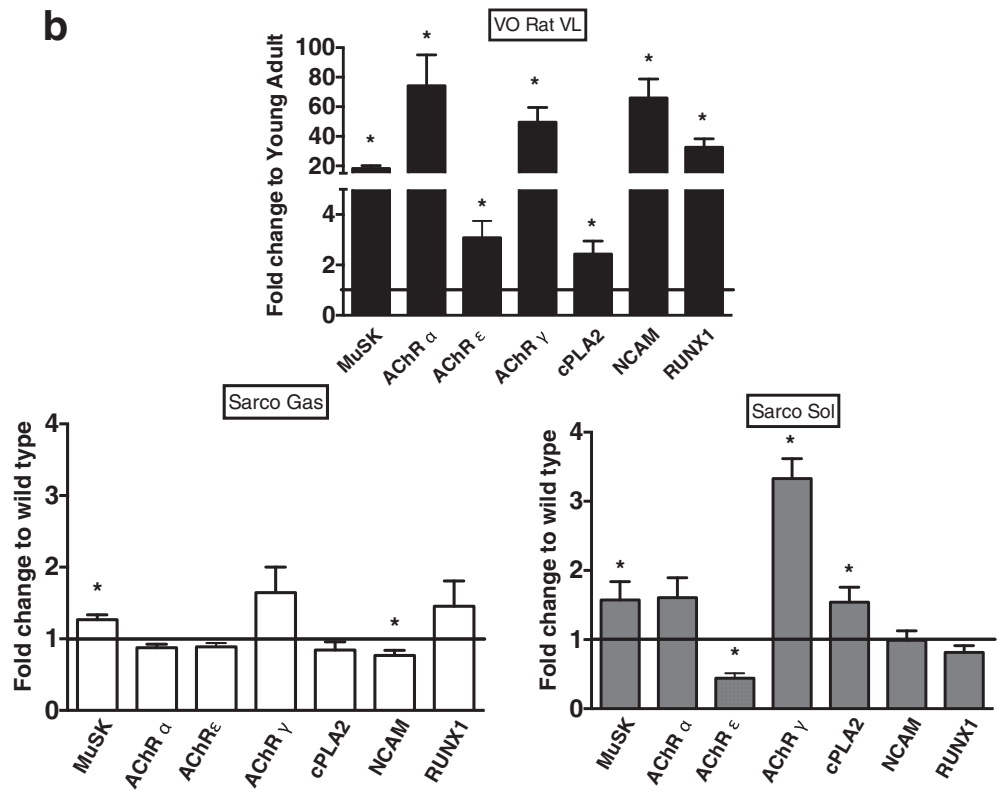

Fig. 3 Morphological and transcriptional markers of denervation. a Abundance of very small fibers in YA vs VO rat vastus lateralis muscle, WT vs Sarco mouse gastrocnemius muscle, and WT vs Sarco mouse soleus muscle. b qPCR analysis of denervation markers: MuSK, AChRa, AChRe, AChRg, cPLA2, NCAM, and RUNX1 in YA $(n=8)$ vs VO $(n=10)$ rat vastus lateralis muscle and WT $(n=8)$ vs Sarco mouse $(n=6)$ gastrocnemius and soleus muscle. TBP was used as endogenous control. Data are shown as means with standard error. $T$ tests were performed. ${ }^{*} P<0.05$ was considered as statistically significant

\section{Transcriptional profile of neurotrophins and related receptors in aged muscle}

The gene expression of neurotrophins (Fig. 4a left panel), including those implicated in promoting axonal growth following nerve injury $[12,13]$, were not significantly elevated in VO muscle, despite the marked accumulation of very small muscle fibers and large increases in expression of denervation-related transcripts. This was also the case in both muscles examined in Sarco mice (Fig. 4b, c left panels), although the denervation stress here is clearly less than with aging and there is likely less requirement for axonal sprouting due to the exclusively post-synaptic nature of the NMJ instability in this model. When considering transcripts for the neurotrophin receptors (Fig. 4b), only neurotrophic tyrosine kinase receptor type 3 (NTRK3; encodes the NT3 receptor) and ciliary neurotrophic factor receptor (CNTFR; encodes the CNTF receptor) were increased in rat VO muscle. The other neurotrophin receptors, p75NTR (encoding the low affinity nerve growth factor receptor for several neurotrophins), neurotrophic tyrosine kinase receptor type 1 (NTRK1; encoding the high-affinity receptor for $N G F)$, neurotrophic tyrosine kinase receptor type 2 (NTRK2; encoding the high-affinity receptor for BDNF), and GDNF family receptor alpha-1 (GFRA1; encoding the GDNF receptor) exhibited no change in rat VO muscle compared to YA muscle (Fig. 3a, c). Similar to aging muscle, there were minimal changes in neurotrophin 

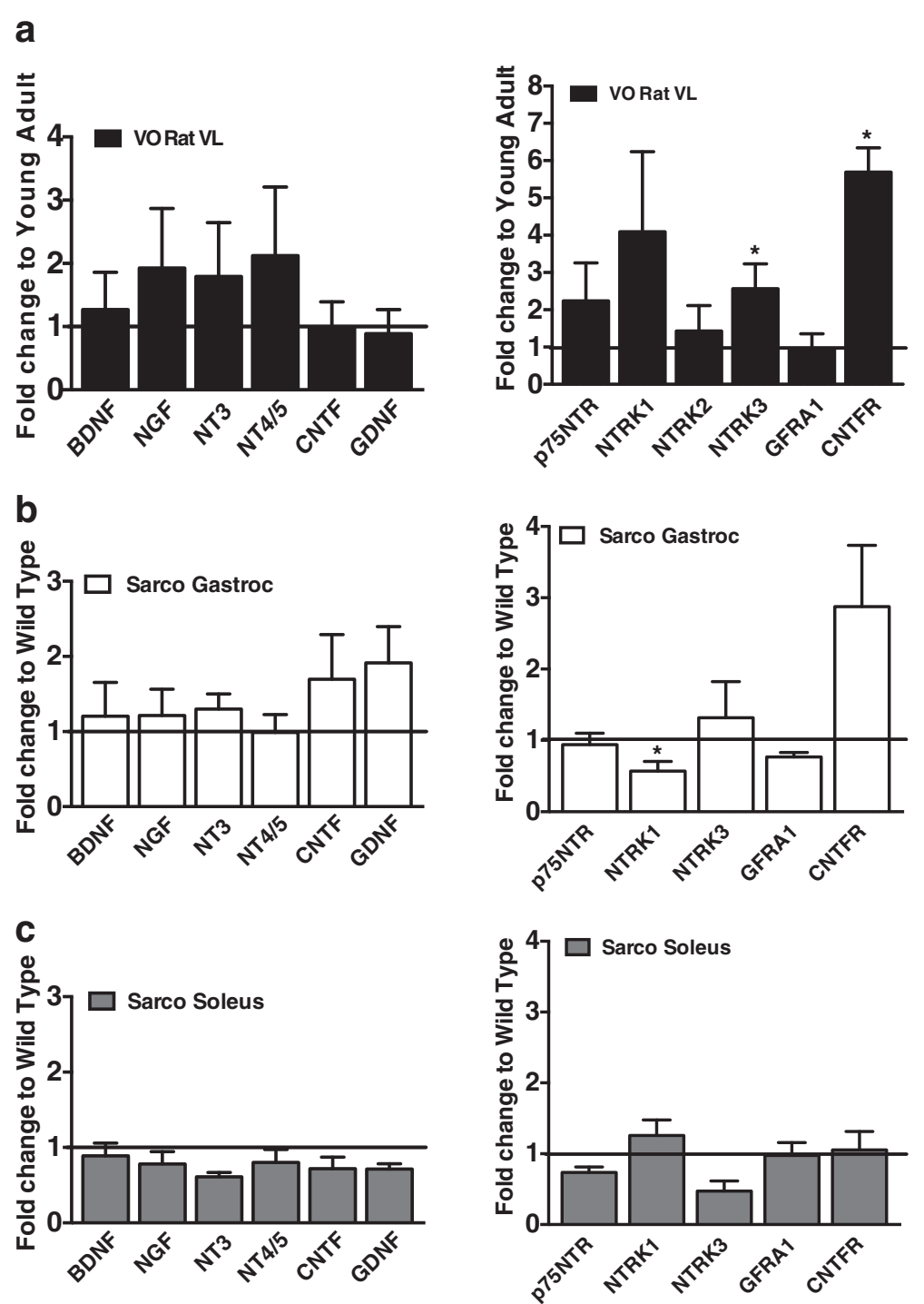

Fig. 4 Neurotrophin and receptor transcript analysis. qPCR analysis of the neurotrophins: BDNF, NGF, NT3, NT4/5, GDNF, CNTF and neurotrophin receptors: $p 75$, NTRK1, NTRK2, NTRK3, GFRA1, and CNTFR in YA $(n=8)$ Vs VO $(n=10)$ rat VL $(\mathbf{a})$, Sarco gastroc $(\mathbf{b})$, and Sarco soleus (c) muscles. TBP was used as endogenous control. Data are shown as means with standard error. $T$ tests were performed. ${ }^{*} P<0.05$ was considered as statistically significant

receptor transcripts in the Sarco mice, with only NTRK1 exhibiting a small decline in expression in Sarco mouse gastrocnemius muscle Fig. 4b, c left panels. Although this analysis does not reveal a decline in neurotrophin signaling in the VO muscle, taken in the context of the large increase in very small fibers and denervation-related transcripts, a reasonable conclusion is that the neurotrophin response is insufficient to facilitate successful reinnervation.

\section{Age-related changes in miRNAs predicted to suppress neurotrophins in aged muscle}

Analysis of the BDNF 3' untranslated region (UTR) using TargetScan (www.targetscan.org) revealed several target sites for BDNF: miR-206-3p, miR-10a-5p, miR-1b, miR-195-5p, and miR-497-5p that were conserved in humans. Both miR-206-3p and miR-1b have the same seed sequence (ACAUUCC). In VO rat muscle, miR-206$3 p$, miR-195-5p, and miR-497-5p were increased significantly. In contrast, miR-1b decreased and there was no change observed in miR-10a-5p (Fig. 5a). Similarly, TargetScan analysis identified the following miRNAs that are conserved in humans and are predicted to influence the NT3 gene: miR-21-5p, miR-222-3p, and miR-221-3p. Both miR-222-3p and miR-221-3p share the same seed sequence (AUGUAGCA). In VO rat muscle, miR-21-5p and miR-221-3p were increased significantly (Fig. 5b). Finally, TargetScan identified the following miRNAs that are 


\section{a}
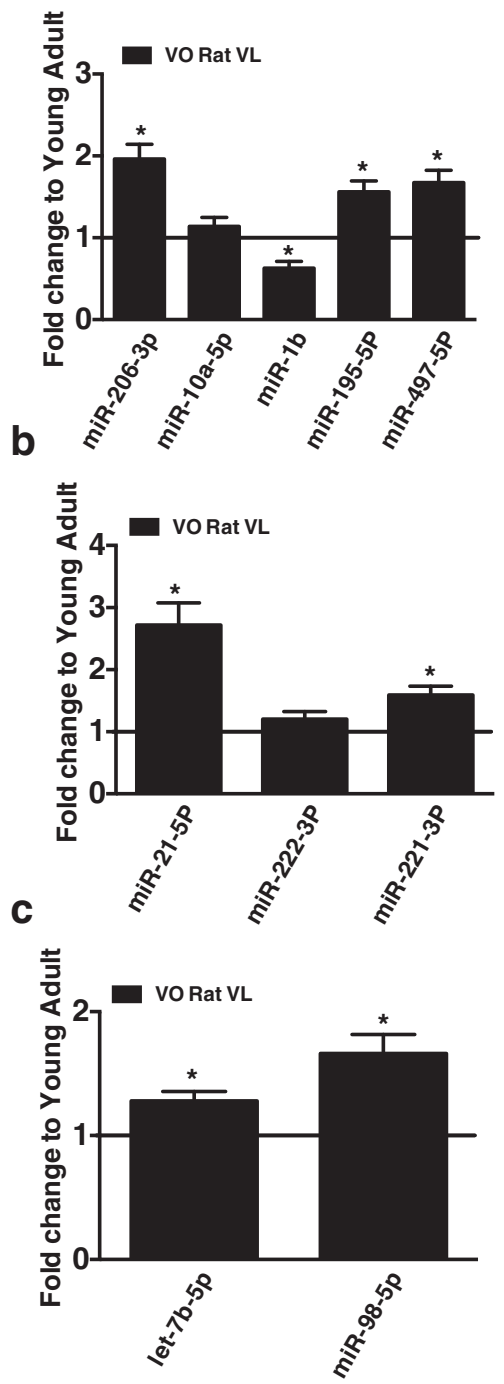
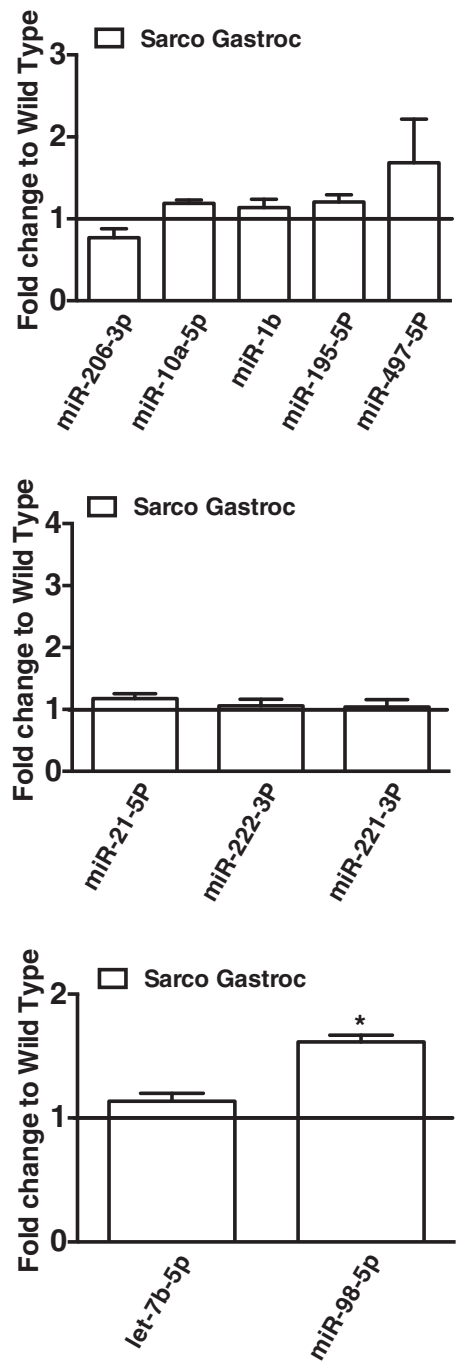

Fig. 5 MicroRNA analysis. MicroRNAs predicted to influence neurotrophins: a BDNF (miR-206-3p, miR-10a-5p, miR-1b, miR-195-5p and miR-497-5p), b NT3 (miR-21-5p, miR-222-3p and miR-221-3p), and c NGF (let-7b-5p and miR-98-5p) were quantified by qPCR analysis in YA $(n=8)$ vs VO $(n=10)$ rat vastus lateralis muscle and WT $(n=8)$ vs Sarco $(n=7)$ gastrocnemius muscle. miR-191a-5p was used as endogenous control. Data are shown as means with standard error. $T$ tests were performed. ${ }^{*} P<0.05$ was considered as statistically significant

conserved in humans as likely to influence NGF transcript levels: let-7b-5p and miR-98-5p and these miRNAs share a common seed sequence (CUACCUCA). Both let-7b-5p and miR-98-5p were increased significantly in $\mathrm{VO}$ rat muscle (Fig. 5c). Thus, collectively, seven out of the ten miRNAs identified as likely candidates for suppressing neurotrophin expression were elevated in aging muscle. Of these, only miR-98-5p exhibited an increase in Sarco mouse gastrocnemius muscle (this analysis was not possible in soleus due to limited tissue).

\section{Discussion}

There is a need for identification of viable therapeutic targets to treat aging muscle in advanced age where the clinical impact of muscle atrophy becomes meaningful. A key step in this endeavor is a clear understanding of the processes that lead to muscle atrophy in advanced age. To this end, the purpose of this study was to evaluate the hypothesis that the accumulation of persistently denervated muscle fibers in advanced age is due to failed reinnervation by comparing changes with aging to changes seen in a mouse model of sporadic denervation, the Sarco mouse.

Our results show that VO rat VL muscle is characterized by marked accumulation of very small fibers and MHC co-expression, two hallmark features of sporadic denervation in aging muscle [3]. Although there are many reasons fiber atrophy could occur, its sporadic nature (atrophied fibers interspersed amongst relatively 
normal sized fibers) in VO muscle is also seen in neuromuscular diseases that exhibit denervation, such as amyotrophic lateral sclerosis and spinal muscular atrophy [22]. Further, to this point, although MHC coexpression can also occur in the context of sedentary lifestyle with aging and is reduced in response to exercise training [23], a distinguishing feature in $\mathrm{VO}$ rat muscle is that MHC co-expression most often colocalizes with other features of denervation, including small and angular fiber size and $\mathrm{Nav}_{1.5}$ expression [3]. Furthermore, VO rat muscle exhibited marked fiber type grouping (indicative of many repeating cycles of denervation and reinnervation [9]) and large induction of transcripts known to respond to denervation. In contrast, despite significant fiber type grouping in Sarco mouse muscle and a high abundance of MHC co-expressing fibers (suggesting that, similar to normal aging, Sarco mice also undergo many repeating cycles of denervation and reinnervation), the abundance of small fibers and induction of denervation-related transcripts compared to WT mice was much smaller than seen with aging. This analysis suggests a robust reinnervation response in young muscle (evidenced by the Sarco mouse) and in turn a poor reinnervation response of aging muscle. In identifying a possible mechanism for failed reinnervation, our analysis suggests that an insufficient neurotrophin response in aging muscle may contribute to the persistence of denervated muscle fibers and that this may be attributable to increases in miRNAs that suppress neurotrophin expression in aging muscle. The translational significance of these observations is that they suggest that up-regulation of neurotrophin signaling could be an effective therapeutic approach for attenuating muscle atrophy by facilitating reinnervation in advanced age where muscle impact is clinically meaningful.

\section{The evolving causes of atrophy in aging muscle}

Whereas at ages $\leq 75$ years old muscle fibers atrophy relatively uniformly [24], in more advanced ages where the severity of muscle atrophy is more likely to have clinical impact [2], the muscle morphology is punctuated by a marked accumulation of small angular fibers that are interspersed amongst relatively normal-looking muscle fibers [4]. Identification of processes that can lead to sporadic fiber atrophy in aging muscle is thus key to understanding the causes of muscle atrophy in advanced age. Although segmental accumulation of mitochondrial defects has been considered a cause of muscle fiber atrophy with aging [25], the fraction of fibers harboring mitochondrial defects that exhibit atrophy is very small $(5 \%)[5,26]$ and thus unlikely to represent a key target. On the other hand, we have shown that there is a marked accumulation of small angular fibers that coincides with the acceleration of muscle atrophy in both slow and fast twitch muscles in advanced aging [5] and that $>90 \%$ of these fibers label positively for a sodium channel isoform, $\mathrm{Nav}_{1.5}$ [3], that is upregulated in response to denervation [27]. It is for this reason that we have focused the current study on identifying mechanisms that could account for the accumulation of persistently denervated muscle fibers in aging muscle.

\section{Sarco mouse as a model of sporadic denervation}

In our studies, we used a genetic model of sporadic denervation, the Sarco mouse [28]. This model was engineered to over-express the endogenous protease, neurotrypsin, in neurons which in turn causes increased agrin cleavage at the neuromuscular junction and reduces activation of MuSK [7]. The result is less recruitment of rapsyn to the subsynaptic microdomain, a resulting loss of cytoskeletal tethering of the postjunctional AChRs, and thus, an increased susceptibility to sporadic denervation. Although we present data from both gastrocnemius and soleus muscles, our analysis shows that for the parameters examined, the changes are generally greater in the soleus muscle. For example, there was a greater increase in fraction of very small muscle fibers and larger changes in denervation-related transcripts in the relatively slow twitch postural soleus muscle compared to the largely fast twitch gastrocnemius muscle in the Sarco mice. Thus, whilst fiber type and muscle recruitment are clearly different between rat VO and mouse Soleus muscles, the Sarco mouse soleus is more affected and might on this basis provide a better indicator of the degree to which reinnervation is compromised with aging.

Consistent with prior studies using this model [7, 28], the Sarco mice exhibited smaller muscle mass and a pronounced fiber type shift punctuated by a large accumulation of MHC co-expressing fibers. Furthermore, phospho-MuSK levels at the NMJ were seen to decrease (Fig. 2a), as would be expected in response to reduced agrin activation of MuSK [7], and this was accompanied by a reduced rapsyn and AChR intensity in the post-synaptic endplate region. In addition, we observed marked fiber type grouping (only examined in soleus muscle) and moderate increases in some denervation-related transcripts (MuSK in both muscles, and $A C h R \gamma$ and $c P L A 2$ in soleus muscle). We also noted a nearly $60 \%$ decrease in $A C h R \varepsilon$ subunit in Sarco soleus muscle, which contrasts with the increase in $A C h R \varepsilon$ that was seen in $\mathrm{VO}$ rat muscle. In this respect, previous studies have shown that following denervation $A C h R \varepsilon$ expression undergoes a multiphasic response: (i) an initial decrease in the first 10 day post-denervation [29]; (ii) a sharp increase observed approximately 4 weeks following denervation; (iii) a decline over the next 4 weeks; and (iv) a gradual secondary increase when denervation persists beyond 2 months [30,31]. Thus, 
the differences between the VO rat and Sarco mouse likely reflect differences in the duration of denervation, with the higher $A C h R \varepsilon$ in VO rat reflecting more prolonged denervation and the reduced $A C h R \varepsilon$ in Sarco mouse reflecting the acute denervation response.

A caveat to comparing the Sarco mouse to aging muscle concerns the frequency of sporadic denervation events. The large increase in frequency of enclosed fibers (Fig. 1c) shows both are exposed to many recurring cycles of denervation and reinnervation. Accepting that it is not possible to obtain a quantitative comparison of the exact frequency of denervation (e.g., on a daily or weekly basis) events in the two models, the value of the Sarco mouse herein is that it provides an indicator of the normal capacity for reinnervation of young adult muscle in the context of significant neuromuscular junction instability, and as such, suggests a compromised reinnervation capacity of $\mathrm{VO}$ muscle. On the other hand, we cannot rule out that the aging muscle might undergo a higher frequency of denervation events, which is itself the problem (rather than failed reinnervation per se). As noted above, however, the $A C h R \varepsilon$ response in the $\mathrm{VO}$ rat is more consistent with prolonged persistent denervation rather than a greater burden of denervation per se.

\section{Causes of denervation and failed reinnervation in aging muscle}

Skeletal muscle undergoes repeating cycles of denervation and reinnervation throughout much of the adult life, resulting in remodeling of the aging motor unit that in turn causes significant grouping of fibers of the same type [9]. The causes of individual denervation events with aging may involve gradual changes in the signaling pathways necessary for NMJ maintenance and/or a sudden change such as muscle injury or motor neuron loss [32]. In our aging rat model, we have previously shown in the same animals studied here that there is a $27 \%$ loss of motor neuron cell bodies in the spinal cord [3], indicating a significant pre-synaptic element causing denervation in $\mathrm{VO}$ rat muscle. This is in contrast to the Sarco mouse where the defect is downstream of the motor neuron and where motor neuron number in the spinal cord is maintained [7]. Thus, although both models exhibit denervation, the causes may be quite different. This notion is supported by the fact that in our study, phospho-MuSK was maintained in aging muscle (decreased in Sarco mouse), rapsyn was increased (reduced in Sarco mouse), and this resulted in only a small loss of AChR intensity in the post-synaptic endplate region of aging muscle (much larger reduction in AChR intensity in Sarco mouse).

Consistent with other studies that have examined transcriptional responses in aging muscle $[19,33]$, in VO rat muscle, we observed increases in several transcripts that change with denervation. The large magnitude of these changes underscores the very significant denervation stress present in the $\mathrm{VO}$ muscle, and this contrasts markedly with the much smaller changes seen in Sarco mouse muscles. Notably, previous studies have shown that the neurotrophins $B D N F, N G F$, and GDNF are important to promote motor axon sprouting and recovery of innervation following surgical denervation $[12,13,34]$. Furthermore, interventions that increase signaling through BDNF and/or its receptor TrkB markedly improve recovery of diaphragm function following cervical spinal cord injury [35-37]. Yet, we observed largely non-significant increases in the neurotrophin and neurotrophin receptor transcripts in VO rat muscle which could indicate a failed response to the marked presence of sporadically denervated fibers in aging muscle. This notion is consistent with previous studies showing impaired BNDF signaling in aging limb muscle [38] and diaphragm [39]. In contrast to aging muscle, the fact that motor neurons are spared in neurotrypsin-overexpressing mice [7] would obligate less extensive axonal sprouting to facilitate reinnervation in this context. Thus, the negligible changes in neurotrophins and their receptors in Sarco mouse muscles are not unexpected. It follows that a significant limitation to our interpretation of the neurotrophin response in VO muscle is that we do not have a model to indicate the "normal" adult neurotrophin response to sporadic motor neuron loss. Such information could be obtained from either experimental models involving a less severe form of denervation involving partial nerve crush or a genetic model involving motor neuron loss. Regardless of this limitation, on the basis of the aforementioned evidence showing the importance of neurotrophins in promoting axonal sprouting and reinnervation following experimental denervation, therapeutically targeting the neurotrophins in advanced age is worthy of evaluation.

\section{Potential involvement of miRNA-induced suppression of neurotrophins}

There has been remarkable progress in recent years in identification of miRNAs, opening up new avenues of research into mechanisms of disease [40, 41], as well as mechanisms that can contribute to muscle impairment in various disorders $[42,43]$. Although it is well established that miRNAs can suppress gene expression by pairing with target mRNAs to direct their degradation or influence the mRNA translation capacity [44], very few studies have examined miRNAs in the context of aging muscle $[45,46]$, and none specifically in the context of the causes of persistent denervation. Thus, in seeking mechanisms that might account for a blunted neurotrophin response to denervation in aging muscle, we first used TargetScan to identify potential miRNAs 
that could suppress neurotrophins. This analysis identified 10 miRNAs that are conserved in humans and which target three neurotrophins involved in promoting axonal sprouting following nerve injury. For $B D N F$, our analysis identified increases in miR-206-3p, miR-195-5p, and miR-497-5p in VO rat muscle. Similarly, for NT3, our analysis identified significant increases in miR-21-5p and miR-221-3P in VO rat muscle. Finally, for NGF, our analysis revealed an increase in both let-7b-5p and miR98-5p in VO rat muscle. Of these, miR-206 has previously been shown to increase in aging mouse muscle [46], consistent with our analysis in VO rat muscle. Similarly, miR-195 (predicted to target $B D N F$ ), and let$7 \mathrm{~b}$ and miR-98 (both predicted to target $N G F$ ) have been shown previously in human muscle to increase with aging [45], consistent with our analysis in VO rat muscle. To our knowledge, none of the other miRNAs that we identified as potentially suppressing neurotrophins have been examined previously in the context of aging muscle.

In contrast to the aforementioned studies in aging muscle, most of the miRNAs studied herein have been examined in the context of experimental denervation, including miR-206 (increases after reinnervation), miR10a-5p (increases four- to seven-fold with denervation), miR-1 (increases up to 10-fold following denervation and remains elevated after reinnervation), miR-195 (increases up to 10-fold with denervation), miR-21 (increases with denervation), miR-221 (no consistent change), miR-222 (no consistent change), and miR-98 (increases up to 10 -fold with denervation) [43, 47, 48]. Note that we also saw an increase in miR-98-5p in Sarco mouse muscle, which likely reflects a normal response to denervation. Thus, the normal response to experimental denervation, and in the current study, a model of NMJ instability (Sarco mouse), is often an increase in miRNAs that are predicted to target neurotrophin genes. The miRNAs that exhibited a different response in VO rat muscle from what is seen with experimental denervation are miR-1, which in experimental denervation increases [48] but in $\mathrm{VO}$ rat muscle was observed to decline, and miR-221, which does not change with denervation [47] but was observed to increase in VO rat muscle. Since miRNAs frequently target more than a single mRNA species, a simple interpretation of the biological significance of the changes observed is challenging. Thus, whether miRNA sponges [41] or other approaches to silencing miRNA signaling would be effective in promoting reinnervation in aging muscle by amplifying the neurotrophin response, will need experimental validation.

\section{Conclusions}

The purpose of our study was to evaluate whether the marked accumulation of persistent denervated fibers in muscle at advanced age is due to failed reinnervation. Consistent with our hypothesis, $\mathrm{VO}$ rat muscle exhibited a much greater accumulation of very small muscle fibers and denervation-related transcripts than Sarco mouse muscle, even though both models evidenced marked fiber type grouping indicative of recurring cycles of denervation and reinnervation. In spite of this significant denervation stimulus in $\mathrm{VO}$ rat muscle, the neurotrophin pathways known to drive axonal sprouting following acute nerve injury exhibited a minimal induction (with most changes not reaching statistical significance). We conclude that failed reinnervation is an important mechanism driving the acceleration of muscle atrophy in advanced age and that therapeutically targeting the neurotrophin response to stimulate axonal sprouting would be worthy of exploration.

\section{Methods}

\section{Experimental procedures}

\section{Animals and tissue collection}

We studied 8-month-old (young adult; YA; $n=8$ ) and 35-36-month-old (very old; VO; $n=10$ ) male Fischer 344 X Brown Norway F1-hybrid (F344BN) rats obtained from the National Institute on Aging (USA) colony. Upon arrival at the University of Calgary Biological Sciences vivarium, the animals were housed individually in cages fitted with filter bonnets and maintained in a 12:12-h light/dark cycle at $23{ }^{\circ} \mathrm{C}$ for at least $48 \mathrm{~h}$ before experiments. On the day of experiments, the animals were anesthetized with $55-65 \mathrm{mg} \mathrm{kg}{ }^{-1}$ of sodium pentobarbital and the left vastus lateralis muscle was removed, trimmed of adipose and connective tissue, and weighed. A slice through the entire midbelly of the vastus lateralis muscle was mounted transversely on pieces of cork using optimal cutting temperature compound and frozen in isopentane pre-cooled in liquid nitrogen before being stored at $-80{ }^{\circ} \mathrm{C}$. Remaining muscle portions were frozen in liquid nitrogen for RNA and microRNA measurements and stored at $-80{ }^{\circ} \mathrm{C}$. All procedures with F344BN rats were conducted with approval from the Animal Care Committee at the University of Calgary (ID B109R-11).

Neurotrypsin over-expressing C57BL/6 mice were provided by Neurotune, Switzerland, and bred at the Research Institute of the McGill University Health Centre vivarium. All of the mice were maintained in a heterozygous state and were backcrossed with wild-type C57BL/6 mice. In all experiments, 8-month-old mice were used to study the impact of sporadic denervation and the fidelity of reinnervation in young adult muscle. On the day of the experiment, the animals were sacrificed with $\mathrm{CO}_{2}$ asphyxiation followed by cervical dislocation. The right soleus and gastrocnemius muscles were dissected free of adipose and connective tissue and weighed, and a portion of each was mounted in tragacanth gum and frozen in isopentane precooled in liquid nitrogen. The remaining portion of each 
muscle was frozen in liquid nitrogen for mRNA and miRNA (gastrocnemius muscle only in Sarco mouse) analyses. All experimental procedures with mice were conducted with approval from the Animal Care Committee at McGill University Health Centre.

\section{Muscle sectioning}

Ten-micrometer-thick cross-sections were cut using a Leica CM-3050-S cryostat $\left(-20{ }^{\circ} \mathrm{C}\right)$ and mounted onto glass slides. The sections were left to air-dry for $2 \mathrm{~h}$ before being stored at $-80{ }^{\circ} \mathrm{C}$ until use. Prior to labeling protocols (see below), the sections were defrosted in slide boxes for $30 \mathrm{~min}$ and allowed to air dry.

\section{Fiber type, size, and proportion assessments}

Muscle cross-sections were immunolabeled for myosin heavy chains (MHCs) I, IIa, IIx, and IIb by a previously described method [24]. The sections were rehydrated with PBS ( $\mathrm{pH} 7.2$ ). These sections were blocked using goat serum (10 \% in PBS) and incubated for $1 \mathrm{~h}$ at room temperature with the following primary antibody solution (unless otherwise stated, from the Developmental Studies Hybridoma Bank, Iowa, USA): mouse IgG2b monoclonal anti-MHC type I (BA-F8, 1:25), mouse IgG1 monoclonal anti-MHC type IIa (SC-71, 1:200), mouse IgM monoclonal anti-MHC type II× (6H1, 1:25), mouse IgM monoclonal anti-MHC type IIb (BF-F3, 1:200), and a rabbit IgG polyclonal anti-laminin (ThermoFisher Scientific). The muscle sections were then washed three times in PBS before being incubated for $1 \mathrm{~h}$ at room temperature with the following secondary antibodies (all obtained from ThermoFisher Scientific): Alexa Fluor 350 IgG2b (y2b) goat anti-mouse (A-21140, 1:500), Alexa Fluor 594 IgG1 (y1) goat anti-mouse (A-21125, 1:100), Alexa Fluor 488 IgM goat anti-mouse (A-21042, 1:500), and Alexa Fluor 488 IgG goat anti-rabbit (A-11008, 1:500) diluted in blocking solution. Due to the presence of four $\mathrm{MHC}$ isoforms in rodent muscle, labeling was performed on two serial sections (section 1: three MHC antibodies + laminin, section 2: one MHC antibody+laminin). Muscle cross-sections were then washed three times in PBS, and coverslips were applied to slides using Prolong Gold (ThermoFisher Scientific; P36930) as mounting medium. The slides were imaged with a Zeiss fluorescence microscope (Axio Imager, Zeiss, Oberkochen, Germany). Frames for analysis were systematically sampled across each muscle section. The frequency of very small fibers was assessed as an index of the degree of persistent denervation in both aging rat and Sarco mice, based upon previous data from our group showing that fibers of this size in VO rat gastrocnemius muscle exhibit $>90 \%$ positive labeling for a marker of denervation, the sodium channel isoform $\mathrm{Nav}_{1.5}$ [3]. Specifically, we used the fiber size corresponding to the first percentile in YA (for rat) or WT (for mice) animals to define very small fiber size. The first percentile was determined by calculating the size distribution of all fibers for all animals in either YA rats or WT mice. In VO rat VL muscle, this corresponded to fibers with a size of $\leq 1000 \mu \mathrm{m}^{2}$, whereas in Sarco mouse, this corresponded to fibers with a size of $650 \mu \mathrm{m}^{2}$ in gastrocnemius muscle and $\leq 575 \mu \mathrm{m}^{2}$ in soleus muscle.

\section{Neuromuscular junction labeling}

We first labeled sections to identify levels of activated MuSK based upon phospho-MuSK staining intensity. Tissue cross-sections of rat VL and mouse gastrocnemius muscle were rehydrated with PBS and then fixed in $2 \%$ paraformaldehyde containing Phosphatase Inhibitor Cocktail tablet for $10 \mathrm{~min}$ at room temperature and then washed $(4 \times 5$ min PBS, $1 \times$ PBS) before being incubated in permeabilization solution ( $0.2 \%$ Tween 20 in PBS) at room temperature for $10 \mathrm{~min}$. The sections were washed again and incubated in blocking solution (10 \% NGS) for 60 min. Incubation with anti-MuSK (phospho Y755) antibody (1:250 in blocking buffer; Rabbit polyclonal, Abcam; ab192583) was performed overnight at $4{ }^{\circ} \mathrm{C}$ in a humidified chamber. The slides were washed and then incubated with AF594 IgG goat anti-rabbit (ThermoFisher Scientific, A11037; 1:100 in blocking buffer) antibody for $1 \mathrm{~h}$ at room temperature. The sections were washed a final time and then mounted with ProLong Gold Antifade Mountant (ThermoFischer Scientific). Images were captured on an Axio Imager M2 (Zeiss) microscope using $\times 40$ objective.

We next examined the downstream impact of alterations in MuSK activation by examining muscle endplate levels of the AChR tethering protein, rapsyn, and AChR intensity levels. Tissue cross-sections of rat VL and mouse gastrocnemius muscle were fixed in acetone for $15 \mathrm{~min}$ at $4{ }^{\circ} \mathrm{C}$ then washed $(2 \times 5$ min PBST, $1 \times$ PBS $)$ before being incubated in permeabilization solution $(0.1 \%$ Triton in PBS) at room temperature for $15 \mathrm{~min}$. The sections were washed again and incubated in blocking solution (1\% NGS $5 \%$ BSA) for 30 min. Incubation with $\alpha$ bungarotoxin 488 conjugate (ThermoFisher Scientific; B13422) and rapsyn (mouse monoclonal, Abcam; Ab11423) was performed at room temperature for $2 \mathrm{~h}$ in a humidified chamber, where all dilutions were made up in blocking solution. The slides were washed, and AF350 IgG1 goat anti-mouse (ThermoFisher Scientific 1:100) was applied for $1 \mathrm{~h}$ at room temperature. The sections were washed a final time and then mounted with ProLong Gold Antifade Mountant (ThermoFischer Scientific). Images were captured on an Axio Imager M2 (Zeiss) and then deconvoluted on Autoquant X3 using the "blind deconvolution" setting and image parameters provided by the Axio imager M2 metadata. Analysis of NMJ labeling was performed using ImageJ (National Institutes of Health, 
Bethesda), with NMJs being traced semi-automatically and the investigator blinded to the group status of the animal.

\section{Gene expression}

Total RNA was extracted from YA and VO rat vastus lateralis muscle samples using the RNeasy Lipid Tissue Mini Kit (Qiagen; 74804). Quantification and purity of total RNA was assessed with a nanodrop by examining the A260/A280 absorption ratio. RNA $(1 \mu \mathrm{g})$ was reverse transcribed to cDNA using qScript ${ }^{\mathrm{Tm}}$ cDNA Synthesis Kit (Quanta biosciences; 95047-025), according to the manufacturer's instructions. Real-time PCR was performed using a StepOnePlus ${ }^{\mathrm{Tw}}$ RealTime PCR system (ThermoFisher Scientific) to quantify the different denervation-related genes (Musk, AChR $\alpha, A C h R \gamma$, cPLA2, NCAM, and RUNX1) (Additional file 1: Table S1) and neurotrophin-related genes (BDNF, NGF, GDNF, CNTF, p75NTR, NTRK1, NTRK2, NTRK3, GFRA1, CNTFR) (Additional file 1: Table S2). TATA box binding protein (TBP) (endogenous control) was obtained from RealTimePrimers.com. Primers were designed with a freely available software, Primer 3 plus. Power SYBR ${ }^{\circ}$ Green PCR Master Mix (ThermoFisher Scientific; 4367659) was used to quantify the mRNA. The thermal profile was $95^{\circ} \mathrm{C}$ for $10 \mathrm{~min}$ and 40 cycles of $95{ }^{\circ} \mathrm{C}$ for $15 \mathrm{~s}$ and $55-60{ }^{\circ} \mathrm{C}$ for $60 \mathrm{~s}$. All real-time PCR experiments were performed in triplicate, and melt curve analysis for each PCR experiment was performed to assess primer dimer formation or contamination. The comparative threshold cycle (CT) method was used to calculate fold changes in expression. Relative foldchanges in gene expression were presented as $2-\Delta \Delta C T$ and normalized to YA (F344BN rats) or WT (Sarco mice) values.

\section{MicroRNA analysis}

Freely available target scan software (www.targetscan.org) was used for predicting microRNAs targeting $B D N F, N T 3$, and NGF genes. Subsequently, we used the software miRBase (www.mirbase.org) to obtain the sequence of the different miRNAs predicted to target these neurotrophin genes. Total RNA was extracted from both YA and VO rat VL muscle using mirVana ${ }^{\text {Tx }}$ miRNA Isolation Kit, with phenol (Thermo Fisher Scientific Inc; AM1560), according to the manufacturers' protocols. MiRNAs were detected using an NCodeTM miRNA qRT-PCR Kit (ThermoFisher Scientific; 45-6612) and real-time PCR (StepOnePlus ${ }^{\text {Tw }}$ Real-Time PCR system, Thermal Cycling Block; ThermoFisher Scientific) with specific primers (Additional file 1: Table S3), Platinum ${ }^{\circ}$ SYBR $^{\circ}$ Green qPCR SuperMix-UDG (ThermoFisher Scientific; 11733-038). All experiments were performed in triplicate. Relative miRNA expression was determined using the CT method, where CT values of individual miRNA data were normalized to CT values of miR-191a-5p. (Fig. 5)

\section{Statistical analysis}

GRAPHPAD PRISM (GraphPad Software, Inc. La Jolla, CA, USA) software was used for statistical analysis. All statistical analyses involved an unpaired two sided $T$ test when comparing two groups. Fiber type distribution was analyzed by two-way ANOVA (fiber type $\mathrm{x}$ group), with a Sidak's post hoc test. The outliers were detected by using GraphPad prism Grubbs' test. $P<0.05$ was considered as statistically significant.

\section{Additional file}

Additional file 1: Table S1. DNA sequences and primers for $\mathrm{GPCR}$ analyses of neurotrophins and neurotrophin receptors in rat skeletal muscle. Table S2. DNA sequences of primers used for qPCR analyses of neurotrophins and neurotrophin receptors in mouse muscle. Table S3. microRNA sequences of primers used to perform QPCR experiments to detect miR levels in rat vastus lateralis and mouse gastrocnemius muscle. (PPTX $1239 \mathrm{~kb})$

\section{Acknowledgements}

This work was supported by operating grants from the Canadian Institutes of Health Research (MOP 119583 and 125986 to R.T. Hepple) and infrastructure support from the Canada Foundation for Innovation (Leaders Opportunity Fund no. 29945 to R.T. Hepple). Dr. S. Spendiff was partially supported by a postdoctoral fellowship from the Research Institute of the McGill University Health Centre. Dr. Stefan Hettwer is an employee of Neurotune (Switzerland).

\section{Authors' contributions}

SA contributed to the experimental design, performed the mRNA and miRNA analyses, and contributed to the writing of the manuscript. SS performed the experiments with aged rats and contributed to the writing of the manuscript. MV conducted the experiments with the Sarco mice and contributed to the writing of the manuscript. DE conducted the immunolabeling experiments in the rat and mouse muscle. AP assisted in the experiments with the Sarco mice. QW conducted the muscle morphology analyses. DM and SH provided expertise with the microRNA analysis and contributed to the writing of the manuscript. SH provided expertise on the Sarco mouse and contributed to the writing of the manuscript. RTH conceived the study, wrote the first draft of the manuscript, and directed the revisions in the revised manuscript. All authors read and approved the final manuscript.

\section{Competing interests}

The authors declare that they have no competing interests.

\section{Author details}

${ }^{1}$ Research Institute of the McGill University Health Centre, EM2.2232, RI MUHC, 1001 Decarie Blvd, Montreal, QC, Canada H4A 3J1. ${ }^{2}$ McGill Research Centre for Physical Activity and Health, McGill University, Montreal, QC, Canada. ${ }^{3}$ Department of Kinesiology and Physical Education, McGill University, Montreal, QC, Canada. ${ }^{4}$ Neurotune, Zurich, Switzerland.

Received: 2 February 2016 Accepted: 5 August 2016

Published online: 01 September 2016

\section{References}

1. Larsson L. Motor units: remodeling in aged animals. J Gerontol A Biol Sci Med Sci. 1995;50:91-5.

2. Cruz-Jentoft AJ, Landi F, Topinkova E, Michel JP. Understanding sarcopenia as a geriatric syndrome. Curr Opin Clin Nutr Metab Care. 2010;13:1-7.

3. Rowan SL, Rygiel K, Purves-Smith FM, Solbak NM, Turnbull DM, Hepple RT. Denervation causes fiber atrophy and myosin heavy chain co-expression in senescent skeletal muscle. PLoS One. 2012;7:e29082.

4. Scelsi R, Marchetti $C$, Poggi P. Histochemical and ultrastructural aspects of $m$. vastus lateralis in sedentary old people (aged 65-89 years). Acta Neuropathol. 1980;51:99-105. 
5. Rowan SL, Purves-Smith FM, Solbak NM, Hepple RT. Accumulation of severely atrophic myofibers marks the acceleration of sarcopenia in slow and fast twitch muscles. Exp Gerontol. 2011;46:660-9.

6. Kulakowski SA, Parker SD, Personius KE. Reduced TrkB expression results in precocious age-like changes in neuromuscular structure, neurotransmission, and muscle function. J Appl Physiol. 2011;111:844-52.

7. Butikofer L, Zurlinden A, Bolliger MF, Kunz B, Sonderegger P. Destabilization of the neuromuscular junction by proteolytic cleavage of agrin results in precocious sarcopenia. Faseb J. 2011;25:4378-93.

8. Gouspillou G, Picard M, Godin R, Burelle Y, Hepple RT. Role of peroxisome proliferator-activated receptor gamma coactivator 1-alpha (PGC-1a) in denervation-induced atrophy in aged muscle: facts and hypotheses. Longevity \& Healthspan. 2013;2:13.

9. Kanda K, Hashizume K. Changes in properties of the medial gastrocnemius motor units in aging rats. J Neurophysiol. 1989;61:737-46.

10. Kang H, Lichtman JW. Motor axon regeneration and muscle reinnervation in young adult and aged animals. J Neurosci. 2013;33:19480-91.

11. Painter MW, Brosius Lutz A, Cheng YC, Latremoliere A, Duong K, Miller CM, Posada S, Cobos EJ, Zhang AX, Wagers AJ, Havton LA, Barres B, Omura T, Woolf CJ. Diminished Schwann cell repair responses underlie age-associated impaired axonal regeneration. Neuron. 2014;83:331-43.

12. Michalski B, Bain JR, Fahnestock M. Long-term changes in neurotrophic factor expression in distal nerve stump following denervation and reinnervation with motor or sensory nerve. J Neurochem. 2008;105:1244-52.

13. Hoyng SA, De Winter F, Gnavi S, de Boer R, Boon LI, Korvers LM, Tannemaat MR, Malessy MJ, Verhaagen J. A comparative morphological, electrophysiological and functional analysis of axon regeneration through peripheral nerve autografts genetically modified to overexpress BDNF, CNTF, GDNF, NGF, NT3 or VEGF. Exp Neurol. 2014:261:578-93.

14. Sartini S, Bartolini F, Ambrogini P, Betti M, Ciuffoli S, Lattanzi D, Di Palma M, Cuppini R. Motor activity affects adult skeletal muscle re-innervation acting via tyrosine kinase receptors. Eur J Neurosci. 2013;37:1394-403.

15. Punga AR, Lin S, Oliveri F, Meinen S, Ruegg MA. Muscle-selective synaptic disassembly and reorganization in MuSK antibody positive MG mice. Exp Neurol. 2011;230:207-17.

16. Ghazanfari N, Morsch M, Reddel SW, Liang SX, Phillips WD. Muscle-specific kinase (MuSK) autoantibodies suppress the MuSK pathway and ACh receptor retention at the mouse neuromuscular junction. J Physiol. 2014:592:2881-97.

17. Urbanchek MG, Picken EB, Kalliainen LK, Kuzon Jr WM. Specific force deficit in skeletal muscles of old rats is partially explained by the existence of denervated muscle fibers. J GerontolA BiolSci MedSci. 2001;56:B191-7.

18. Rieger F, Nicolet M, Pincon-Raymond M, Murawsky M, Levi G, Edelman GM. Distribution and role in regeneration of N-CAM in the basal laminae of muscle and Schwann cells. J Cell Biol. 1988:107:707-19.

19. Ibebunjo C, Chick JM, Kendall T, Eash JK, Li C, Zhang Y, Vickers C, Wu Z, Clarke BA, Shi J, Cruz J, Fournier B, Brachat S, Gutzwiller S, Ma Q, Markovits J, Broome M, Steinkrauss M, Skuba E, Galarneau JR, Gygi SP, Glass DJ. Genomic and proteomic profiling reveals reduced mitochondrial function and disruption of the neuromuscular junction driving rat sarcopenia. Mol Cell Biol. 2013;33:194-212

20. Wang X, Blagden C, Fan J, Nowak SJ, Taniuchi I, Littman DR, Burden SJ. Runx1 prevents wasting, myofibrillar disorganization, and autophagy of skeletal muscle. Genes Dev. 2005;19:1715-22.

21. Bhattacharya A, Muller FL, Liu Y, Sabia M, Liang H, Song W, Jang YC, Ran Q, Van Remmen H. Denervation induces cytosolic phospholipase A2-mediated fatty acid hydroperoxide generation by muscle mitochondria. J Biol Chem. 2009;284:46-55.

22. Baloh RH, Rakowicz W, Gardner R, Pestronk A. Frequent atrophic groups with mixed-type myofibers is distinctive to motor neuron syndromes. Muscle Nerve. 2007;36:107-10.

23. Williamson DL, Godard MP, Porter DA, Costill DL, Trappe SW. Progressive resistance training reduces myosin heavy chain coexpression in single muscle fibers from older men. J Appl Physiol. 2000;88:633.

24. Gouspillou G, Sgarioto N, Kapchinsky S, Purves-Smith F, Norris B, Pion CH, Barbat-Artigas S, Lemieux F, Taivassalo T, Morais JA, Aubertin-Leheudre M, Hepple RT. Increased sensitivity to mitochondrial permeability transition and myonuclear translocation of endonuclease $\mathrm{G}$ in atrophied muscle of physically active older humans. Faseb J. 2014;28:1621-33.

25. Wanagat J, Cao Z, Pathare P, Aiken JM. Mitochondrial DNA deletion mutations colocalize with segmental electron transport system abnormalities, muscle fiber atrophy, fiber splitting, and oxidative damage in sarcopenia. Faseb J. 2001;15:322-32.
26. Bua E, Johnson J, Herbst A, Delong B, McKenzie D, Salamat S, Aiken JM. Mitochondrial DNA-deletion mutations accumulate intracellularly to detrimental levels in aged human skeletal muscle fibers. Am J Hum Genet. 2006;79:469-80.

27. Yang JS, Sladky JT, Kallen RG, Barchi RL. TTX-sensitive and TTX-insensitive sodium channel mRNA transcripts are independently regulated in adult skeletal muscle after denervation. Neuron. 1991;7:421-7.

28. Vrijbloed JW, Hettwer S, Kucsera S, Fariello RG. Animal model for agrindependent sarcopenia: the Sarco mouse. Journal of Nutrition, Health \& Aging. 2011;15:A500.

29. Rimer M, Mathiesen I, Lomo T, McMahan UJ. gamma-AChR/epsilon-AChR switch at agrin-induced postsynaptic-like apparatus in skeletal muscle. Mol Cell Neurosci. 1997;9:254-63.

30. Zhang Y, Liu A, Zhang W, Jiang H, Cai Z. Correlation of contractile function recovery with acetylcholine receptor changes in a rat muscle flap model. Microsurgery. 2010;30:307-13.

31. Adams L, Carlson BM, Henderson L, Goldman D. Adaptation of nicotinic acetylcholine receptor, myogenin, and MRF4 gene expression to long-term muscle denervation. J Cell Biol. 1995;131:1341-9.

32. Hepple RT, Rice CL. Innervation and neuromuscular control in ageing skeletal muscle. J Physiol. 2016;594:1965-78.

33. Barns M, Gondro C, Tellam RL, Radley-Crabb HG, Grounds MD, Shavlakadze T. Molecular analyses provide insight into mechanisms underlying sarcopenia and myofibre denervation in old skeletal muscles of mice. Int J Biochem Cell Biol. 2014;53:174-85.

34. Zhao C, Veltri K, Li S, Bain JR, Fahnestock M. NGF, BDNF, NT-3, and GDNF mRNA expression in rat skeletal muscle following denervation and sensory protection. J Neurotrauma. 2004;21:1468-78.

35. Gransee HM, Zhan WZ, Sieck GC, Mantilla CB. Targeted delivery of TrkB receptor to phrenic motoneurons enhances functional recovery of rhythmic phrenic activity after cervical spinal hemisection. PLoS One. 2013;8:e64755.

36. Mantilla CB, Gransee HM, Zhan WZ, Sieck GC. Motoneuron BDNF/TrkB signaling enhances functional recovery after cervical spinal cord injury. Exp Neurol. 2013;247:101-9.

37. Gransee HM, Zhan WZ, Sieck GC, Mantilla CB. Localized delivery of brainderived neurotrophic factor-expressing mesenchymal stem cells enhances functional recovery following cervical spinal cord injury. J Neurotrauma. 2015;32:185-93.

38. Personius KE, Parker SD. TrkB expression at the neuromuscular junction is reduced during aging. Muscle Nerve. 2013;47:532-8.

39. Greising SM, Stowe JM, Sieck GC, Mantilla CB. Role of TrkB kinase activity in aging diaphragm neuromuscular junctions. Exp Gerontol. 2015;72:184-91.

40. Stenvang J, Petri A, Lindow M, Obad S, Kauppinen S. Inhibition of microRNA function by antimiR oligonucleotides. Silence. 2012;3:1.

41. Tay FC, Lim JK, Zhu H, Hin LC, Wang S. Using artificial microRNA sponges to achieve microRNA loss-of-function in cancer cells. Adv Drug Deliv Rev. 2015; 81:117-27.

42. Russell AP, Wada S, Vergani L, Hock MB, Lamon S, Leger B, Ushida T, Cartoni R, Wadley GD, Hespel P, Kralli A, Soraru G, Angelini C, Akimoto T. Disruption of skeletal muscle mitochondrial network genes and miRNAs in amyotrophic lateral sclerosis. Neurobiol Dis. 2012;49C:107-17.

43. Soares RJ, Cagnin S, Chemello F, Silvestrin M, Musaro A, De Pitta C, Lanfranchi $\mathrm{G}$, Sandri M. Involvement of microRNAs in the regulation of muscle wasting during catabolic conditions. J Biol Chem. 2014;289:21909-25.

44. Fabian MR, Sonenberg N, Filipowicz W. Regulation of mRNA translation and stability by microRNAs. Annu Rev Biochem. 2010;79:351-79.

45. Drummond MJ, McCarthy JJ, Sinha M, Spratt HM, Volpi E, Esser KA, Rasmussen BB. Aging and microRNA expression in human skeletal muscle: a microarray and bioinformatics analysis. Physiol Genomics. 2011:43:595-603.

46. Valdez G, Heyer MP, Feng G, Sanes JR. The role of muscle microRNAs in repairing the neuromuscular junction. PLoS One. 2014;9:e93140.

47. Jeng SF, Rau CS, Liliang PC, Wu CJ, Lu TH, Chen YC, Lin CJ, Hsieh CH. Profiling muscle-specific microRNA expression after peripheral denervation and reinnervation in a rat model. J Neurotrauma. 2009:26:2345-53.

48. Hsieh CH, Jeng SF, Wu CJ, Lu TH, Yang JC, Chen YC, Lin CJ, Rau CS. Altered expression of the microRNAS and their potential target genes in the soleus muscle after peripheral denervation and reinnervation in rats. J Trauma. 2011;70:472-80 